Military Technical College Kobry El-Kobbah, Cairo, Egypt

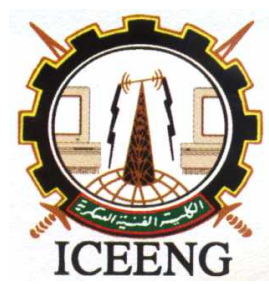

\author{
$6^{\text {th }}$ International Conference \\ on Electrical Engineering \\ ICEENG 2008
}

\title{
A new model of radar signal processor based on self-adaptive band-pass filter
}

\author{
By
}

\section{K.H.Moustafa*}

\section{$\underline{\text { Abstract: }}$}

Self-adaptive band-pass filters are devices, which produce a band-pass filter characteristic around a signal carrier frequency. In this paper a new model of these filters is build based on [1].

This filter has the ability to select rapidly the carrier frequency from a modulated signal. When a train of radio frequency pulses fully immersed in a high-level noise excites this filter, it tracks the entire band and extracts the carrier frequency. The self-adaptive bandpass filter operates as an ECCM (Electronic Counter-Countermeasures) technique because it is trying to cancel out the effects of jammers especially those with barrage jamming over the entire radar band, or spot jamming in which the noise power is concentrated into the radar receiver bandwidth [2,3]. This ECCM technique is applied to the RF section at the radar receiver.

\section{Keywords:}

Self adaptive filter and ECCM. 
In [1] Rhodes showed that if the instantaneous frequency of a signal instead of the signal itself is considered, then devices which perform non-linear operations could perform linear operation on the instantaneous frequency of the signal. Hence transfer functions may be defined directly relating the output to the input instantaneous frequency. In this paper a new model of self-adaptive band-pass filter is build based on this idea.

The performance of this filter is measured in three ways. First, when the filter is excited by a single carrier frequency combined with a noise, it tracks the entire band and selects the carrier frequency signal. Second, when the input is a train of radio frequency pulses fully immersed in noise, which simulate radar echo under barrage jamming, the filter tracks the entire band and extracts the RF pulses with the correct pulse width and pulse repetition period. Third, when the input is a train of radio frequency pulses combines with a high-level band limited noise, which simulate radar echo under spot jamming, the filter tracks the radar receiver band and extracts the RF pulses with the correct parameters as before.

\section{Self-Adaptive Band-Pass Filter:}

The self-adaptive band-pass filter shown in Figure(1) is a linear frequency network that has an instantaneous input frequency $w_{1}(t)$. The Laplace transform of the input frequency is $w_{1}(s)$ which is then split two ways. One way is applied to the first input of an upper side-band mixer, and the other is applied to a delay line, which is then connected, to the other input of the mixer. If $\tau$ is the delay of the delay line then the transforms of the signals applied to the mixer are $w_{1}(s)$ and $\left[\mathrm{Z} w_{1}(s)\right]$ with the resulting output:

$$
(1+Z) W_{l}(S)
$$

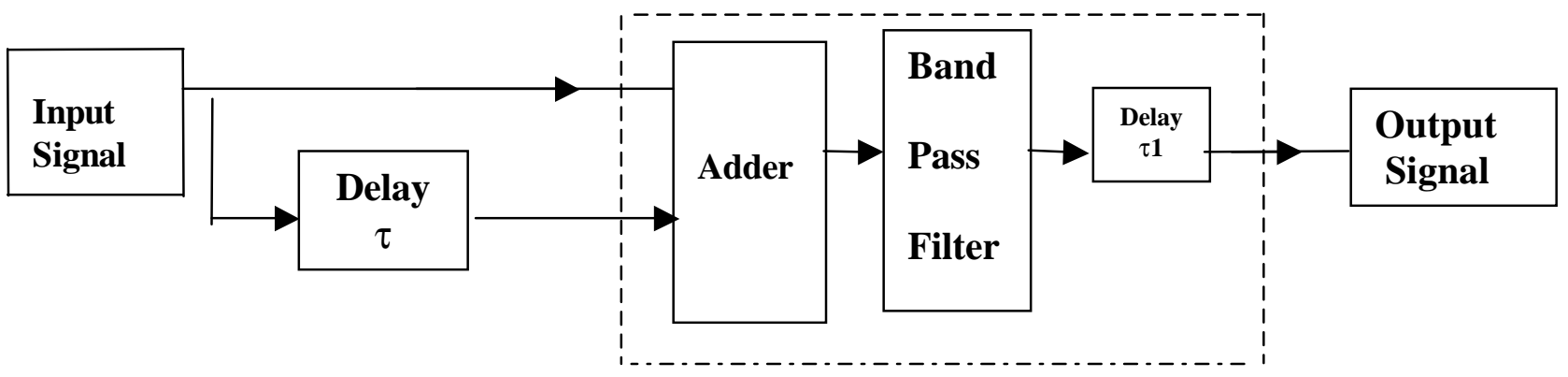

Upper band mixer

Figure (1): Main block diagram of self-adaptive band-pass filter 
Consider the delay $\tau_{1}$ on the output of the mixer due to the band-defining filter. Then, we have $\quad H\left(z, z_{1}\right)=(1+z) z_{1}$ Hence $\quad\left|H\left(z, z_{1}\right)\right|_{P=j \Omega}^{2}=\cos ^{2}(\tau \cdot \Omega)$

This filter has a Quasi-low-pass characteristic. The mixer is a three port device with two inputs $W_{l}(t)$ and $W_{2}(t)$. For the upper side-band mixer we have an output

$$
w_{0}(t)=w_{1}(t)+w_{2}(t)
$$

This requires band filtering as shown in Figure(1) to remove unwanted sidebands producing an associated delay. Furthermore, it should be noted that no output occurs unless signals are present at both input ports. Figure(2) shows the representation of the upper side-band mixer using SIMULINK.

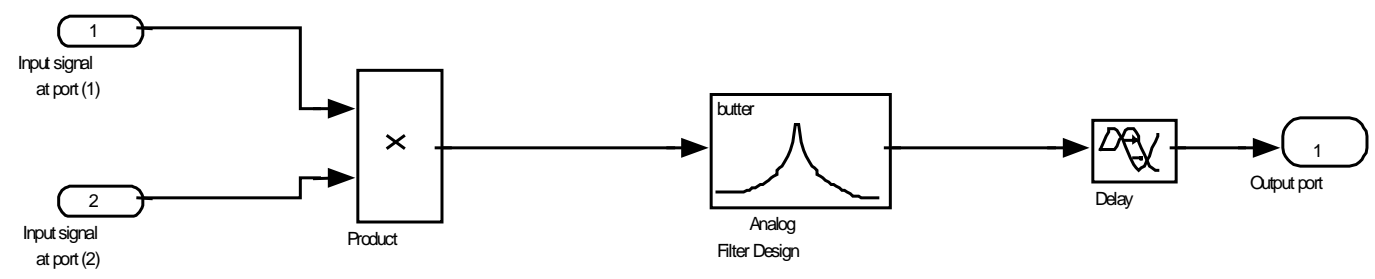

Figure (2): Representation of the upper side-band mixer using SIMULINK

In this case the output of self-adaptive band-pass filter is compared with the output of a conventional band-pass filter, which has a wide bandwidth around the desired radar central frequency to show the excellent capability of self-adaptive band-pass filter in extracting the carrier frequency. Self-adaptive band-pass filter is shown in Figure(3)

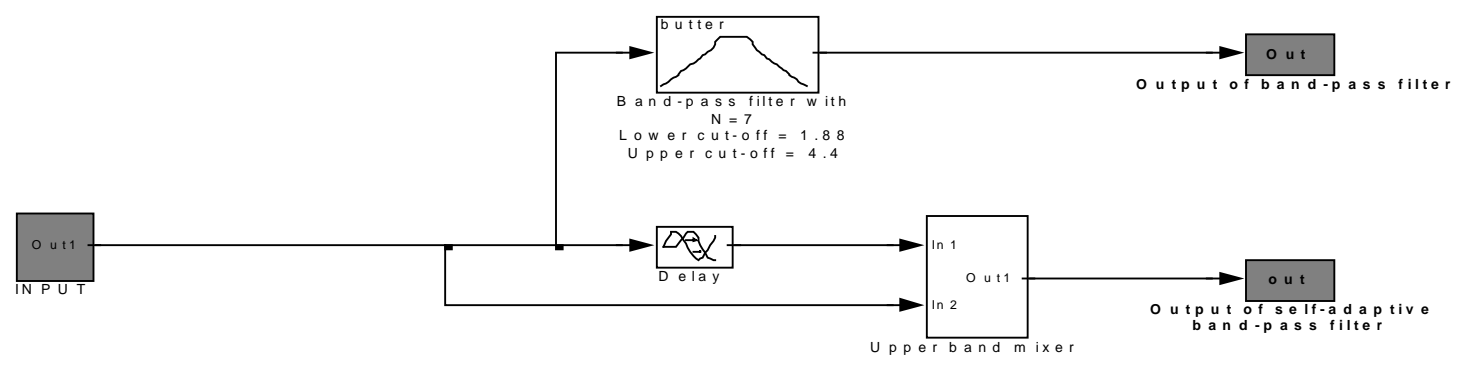

Figure (3): Representation of Self-adaptive band-pass filter using SIMULINK 
The filter is excited by a signal described as:

$A \times \sin \left(2 \times \pi \times f_{0}+\right.$ phase as a function of noise $)$

$5 \times \sin (2 \times \pi \times 0.5 G H z+(1.4) \times$ noise $)$

Figure(4) shows the input signal at both frequency and time domains.

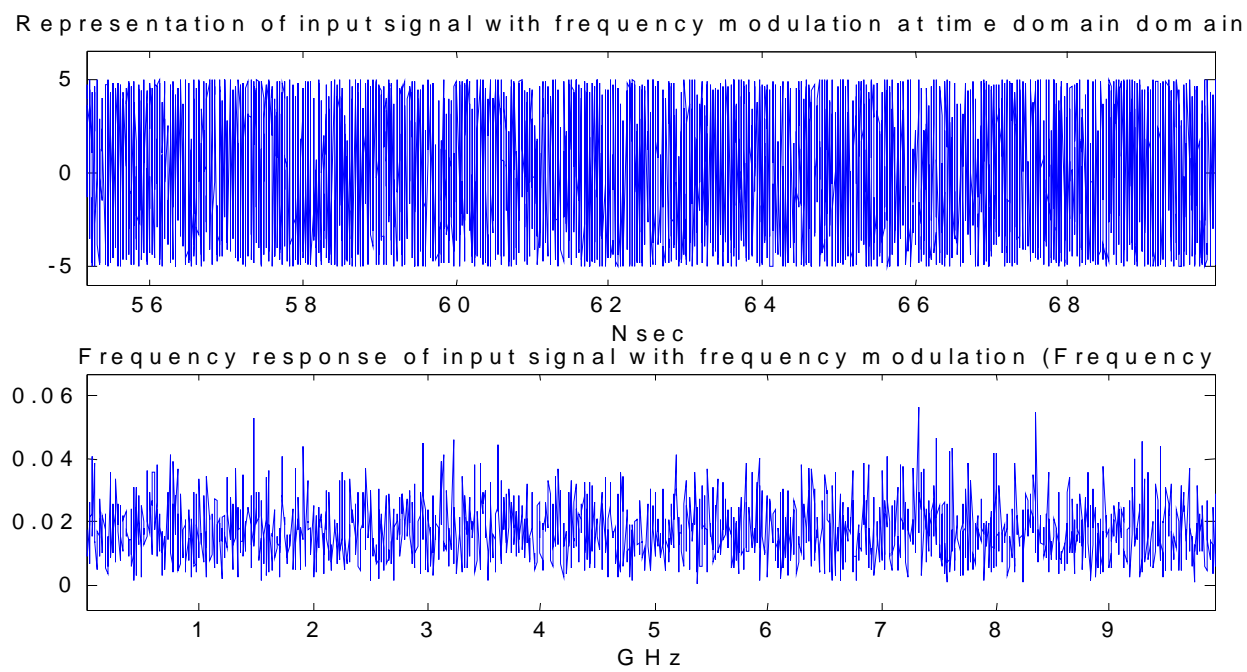

Figure(4): Representation of input signal at frequency and time domains

From the above figure, it is clear that the input signal is fully immersed with a very high level noise. We study the effect of the value of delay shown in Figure(3) for tracking the entire bands and extract the carrier frequency. Figure(5) shows a comparison of the frequency response of self-adaptive band-pass filter at delays 0.001, 0.1, 3 and $7 \mathrm{nsec}$ respectively. The best result is occurred at delay of $7 \mathrm{nsec}$.
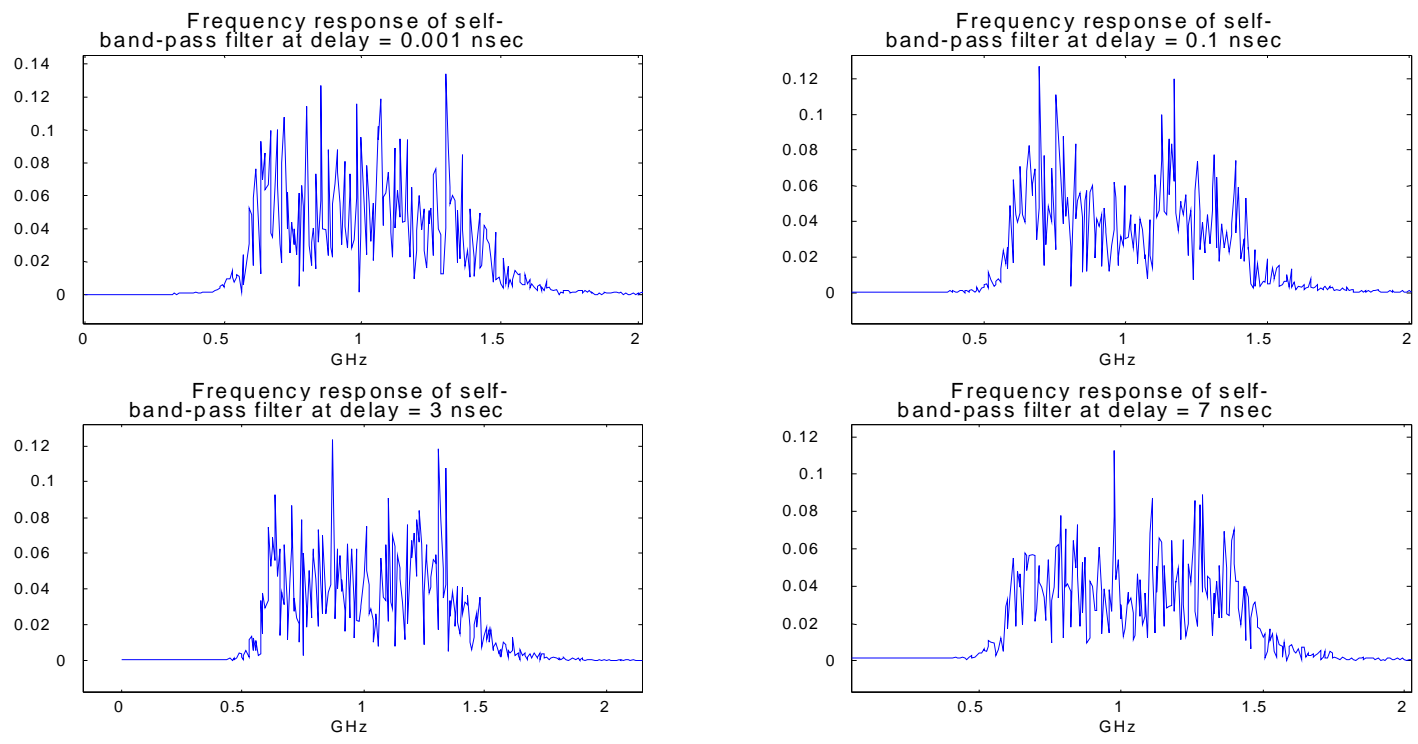

Figure(5): Effect of delay time shown in Figure(3) 
Figure(6) shows a comparison between the output of self-adaptive band-pass filter at best delay ( $7 \mathrm{nsec}$ ) and conventional one.
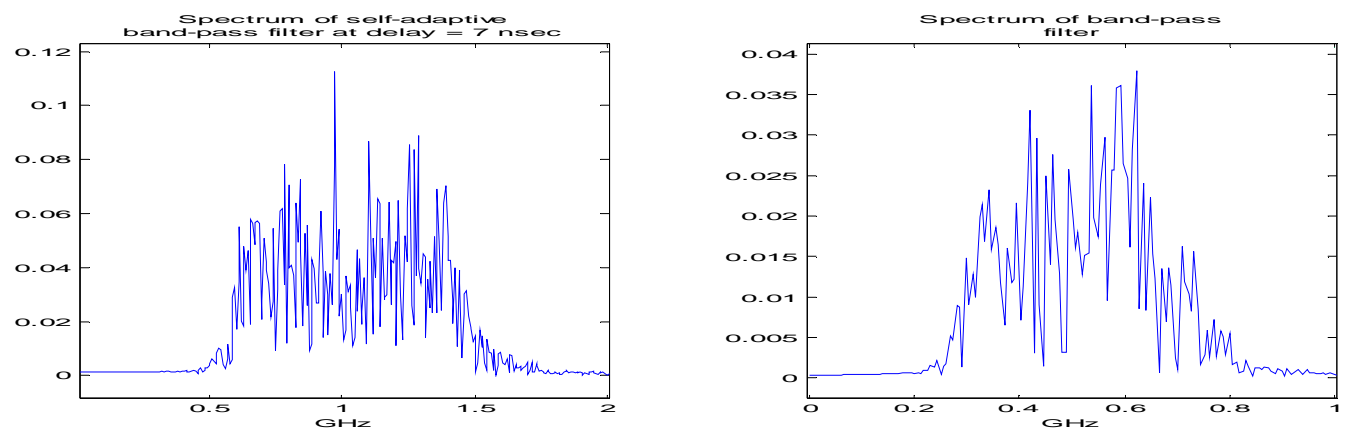

Figure(6): Comparison between the output of self-adaptive band-pass filter and conventional band-pass filter

From the above figure, it is clear that the self-adaptive band-pass filter is successfully in tracking the entire band and extracting the carrier frequency while the conventional one is failed.

\section{Self-Adaptive Band-Pass Filter As ECCM Technique:}

The objective of an ECM (Electronic Countermeasures) system are either to deny the radar an opportunity to detect a target or to deceive the radar into following either the wrong target (such as a chaff cloud) or a non-existent target that has been generated electronically. Two methods of denial are barrage and spot jamming. The self-adaptive band-pass filter is capable to counter these types of jamming.

\section{a) Self-Adaptive Band-Pass Filter As ECCM Technique against Barrage Jamming:}

In this case a train of radar pulses fully immersed in a high-level noise, which simulates radar echo under barrage jamming, excites the self-adaptive band-pass filter. The radar echo has the following parameters; central frequency $f_{0}=0.5 \mathrm{GHz}$, pulse width $\delta=10$ $\mathrm{nsec}$, and pulse repetition period $(\mathrm{PRP})=60 \mathrm{nsec}$. The simulation of the above input is shown at Figure(7).

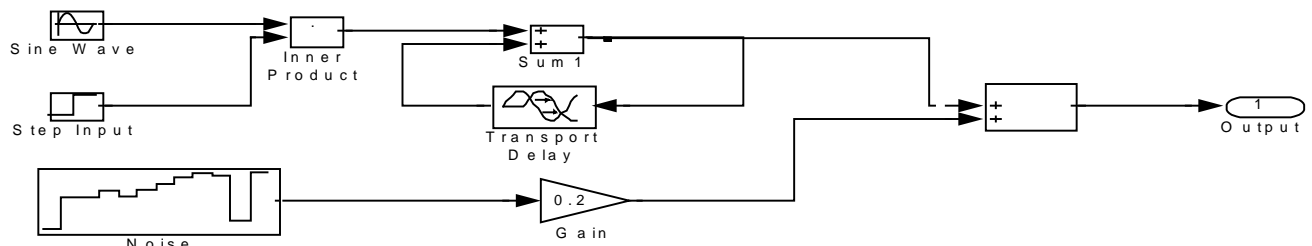

Figure(7): Simulation of radar echo under barrage jamming 
Representation of the radar echo at clear condition, radar echo with barrage jamming, and output from the desired filter are shown respectively at Figure(8).
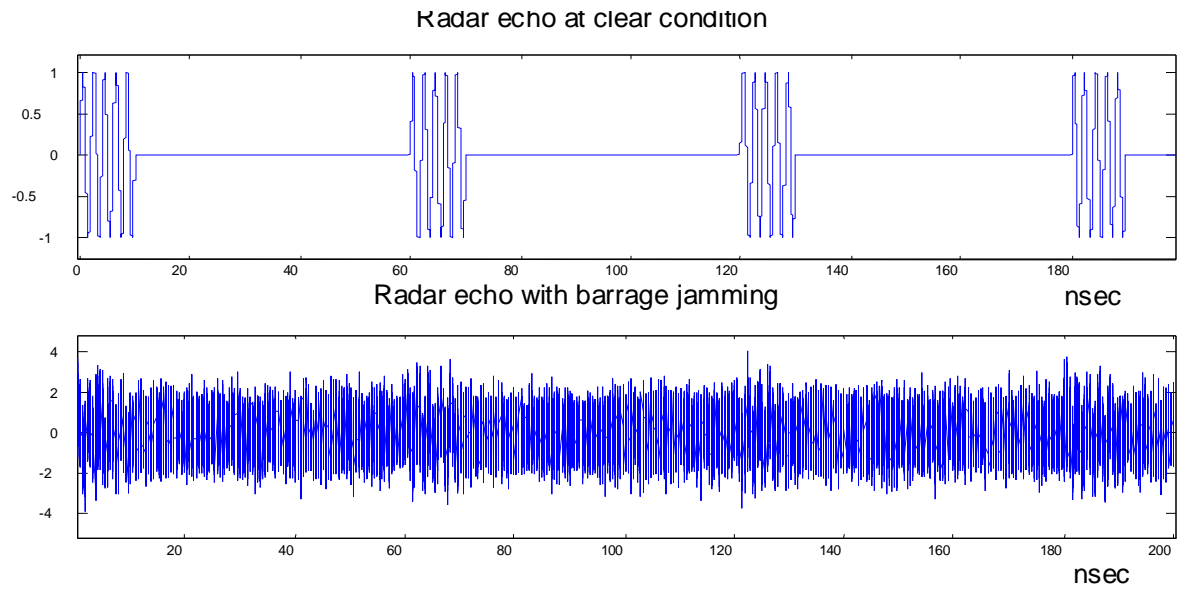

Output of self-adaptive band-pass filter at delay $=0.1 \mathrm{nsec}$
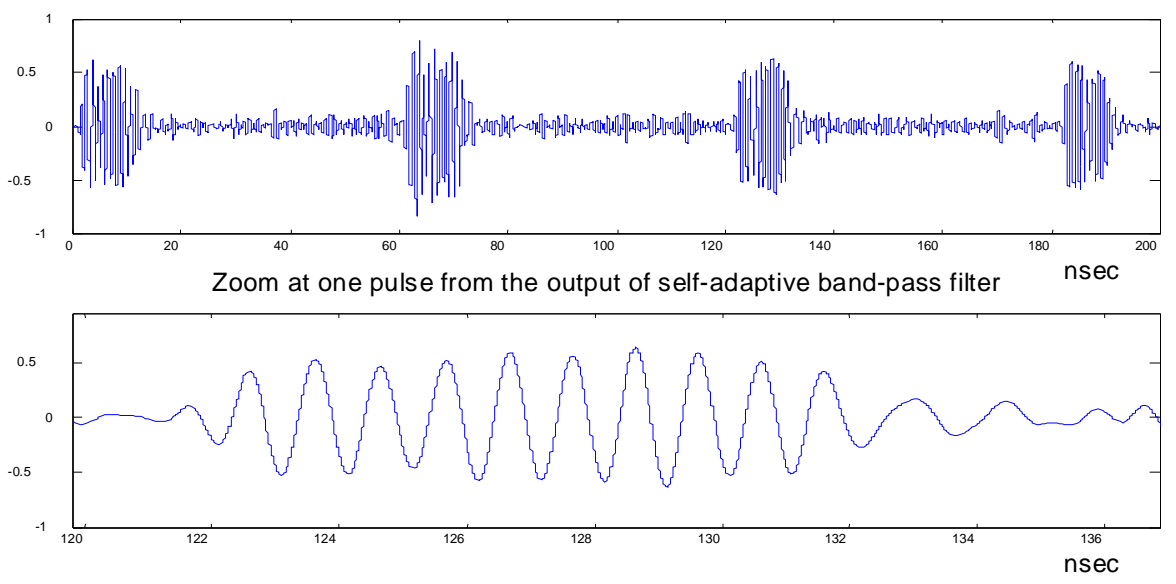

Figure(8): Representation of the radar echo at clear condition, radar echo with barrage jamming, and output from the desired filter respectively.

In Figure(8), radar echo is fully immersed with a barrage jamming. Self adaptive-bandpass filter is successfully in tracking the entire band and extracting the RF pulses with the correct pulse width and pulse repetition period.

\section{b) Self-Adaptive Band-Pass Filter As ECCM Technique against Spot Jamming:}

In this case a train of radio frequency pulses combines with a high-level band noise, which simulate radar echo under spot jamming, excites the self-adaptive band-pass filter. The radar echo has the same parameters as before. The simulation of this input is shown in Figure(9). 


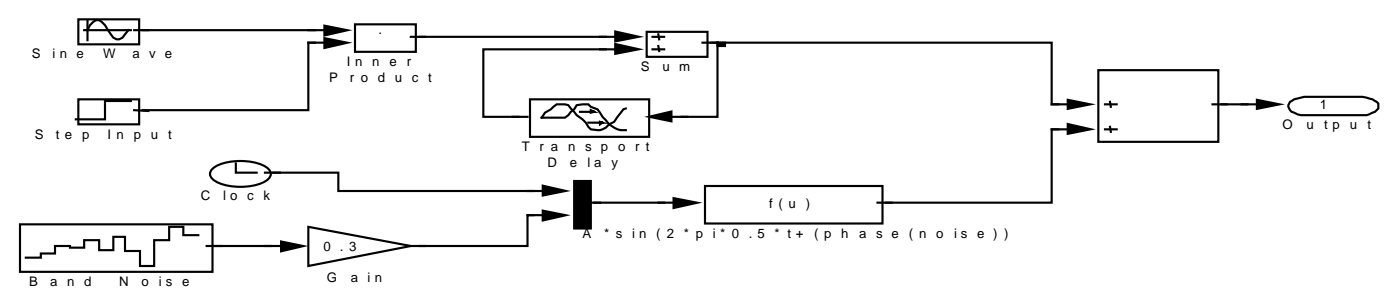

Figure(9): Simulation of radar echo under spot jamming

Representation of the radar echo at clear condition, radar echo with spot jamming, and output from self-adaptive band-pass filter at best delay $(0.23 \mathrm{nsec})$ are shown respectively at Figure(10).
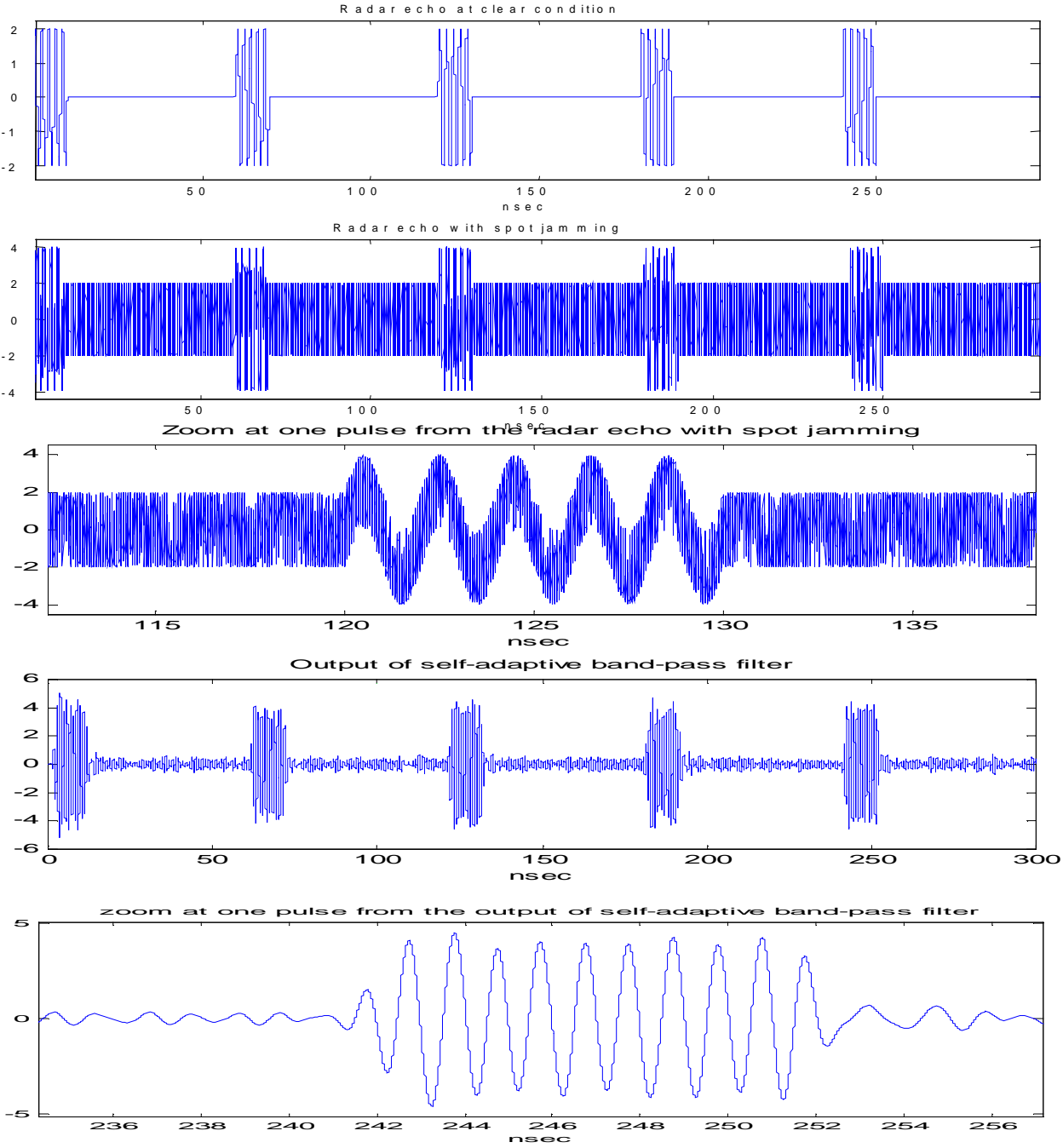

Figure(10): Representation of the radar echo at clear condition, radar echo with spot jamming, and output from self-adaptive band-pass filter at delay $=0.23 \mathrm{nsec}$ respectively. 
As shown in Figure(10), self-adaptive band-pass filter is also successfully in tracking the radar receiver band and extracting RF pulses with the correct pulse width and pulse repetition period as before.

\section{Conclusion:}

A new model of self-adaptive band-pass filter is introduced. This filter has the ability to select rapidly the carrier frequency from a signal fully immersed with a high level noise. When a train of radio frequency pulses fully immersed in a high-level noise excites this filter, it tracks the entire band and extracts the radio frequency pulses again. So, the selfadaptive band-pass filter operates as an ECCM (Electronic Counter- Countermeasures) technique. This ECCM technique is related to RF section at the radar receiver against both barrage and spot jamming.

\section{References:}

[1] J.D. RHODES, "LINEAR FREQUENCY NETWORKS WITH APPLICATIONS TO FREQUENCY SET-ON OSCILLATORS AND SELF-ADAPTIVE BANDPASS FILTERS," International Journal of Circuit Theory and Applications, Vol.18, pp.121-134, 1990.

[2] Leroy B.VanBrunt, “Applied ECM, volume 2,” United States of America, July, 1985.

[3] M.V.Maksimov, M.P. Bobnev, L.N. Shustov, B.K. Krivitskiy, G.I. Gorgonov, V.A.I1'in, and B.M. Stepanov, "RADAR ANTI-JAMMING TECHNIQUES," Artech House, Inc., 1979. 